\title{
Monolithic polymeric porous superhydrophobic material with pneumatic plastron stabilization for functionally durable drag reduction in blood- contacting biomedical applications
}

\author{
Jennifer Marlena', Justin Kok Soon Tan', Zenggan Lin', David Xinzheyang Li², Boxin Zhao ${ }^{3}$, Hwa Liang Leo', \\ Sangho $\mathrm{Kim}^{1}$ and Choon Hwai Yap (1) ${ }^{4}$
}

\begin{abstract}
Superhydrophobic (SHP) surfaces can provide substantial reductions in flow drag forces and reduce blood damage in cardiovascular medical devices. However, strategies for functional durability are necessary, as many SHP surfaces have low durability under abrasion or strong fluid jetting or eventually lose their air plastron and slip-flow capabilities due to plastron gas dissolution, high fluid pressure, or fouling. Here, we present a functional material that extends the functional durability of superhydrophobic slip flow. Facile modification of a porous superhydrophobic polytetrafluoroethylene (PTFE, Teflon) foam produced suitable surface structures to enable fluid slip flow and resist protein fouling. Its monolithic nature offered abrasion durability, while its porosity allowed pressurized air to be supplied to resist fluid impalement and to replenish the air plastron lost to the fluid through dissolution. Active pore pressure control could resist high fluid pressures and turbulent flow conditions across a wide range of applied pressures. The pneumatically stabilized material yielded large drag reductions (up to 50\%) even with protein fouling, as demonstrated from high-speed water jetting and closed loop pressure drop tests. Coupled with its high hemocompatibility and impaired protein adsorption, this easily fabricated material can be viable for incorporation into blood-contacting medical devices.
\end{abstract}

\section{Introduction}

Blood-contacting medical devices such as indwelling intravenous catheters, implants (e.g., stents and heart valves), and extracorporeal circuits (e.g., cardiopulmonary bypass circuits, extracorporeal membrane oxygenator, etc. $)^{1}$ are commonly associated with deleterious patient outcomes stemming from device-induced thrombosis ${ }^{2,3}$ and hemolysis induced by excessive stresses on blood., ${ }^{4,5}$ For example, up to $10 \%$ of patients per year encounter

\footnotetext{
Correspondence: Choon Hwai Yap (c.yap@imperial.ac.uk)

'Department of Biomedical Engineering, National University of Singapore,

Singapore, Singapore

${ }^{2}$ Department of Civil \& Environmental Engineering, University of Alberta,

Edmonton, $\mathrm{AB}$, Canada

Full list of author information is available at the end of the article

These authors contributed equally: Jennifer Marlena, Justin Kok Soon Tan
}

complications after being fitted with ventricular assist devices, accounting for up to $51.3 \%$ of readmissions ${ }^{6}$. Superhydrophobic (SHP) materials have received growing attention for their suitability to address these problems ${ }^{7-9}$ by enabling fluid slip and reduction of fluid stresses and by preventing coagulation reactions.

SHP surfaces have low surface energies and textured surfaces that reduce flow friction and $\mathrm{drag}^{7,10}$ to reduce stress-induced blood damage and hemolysis. Micro- or nanoscale surface asperities trap pockets of air (air plastrons), which reduce liquid-solid contact and hence the accompanying skin friction. This reduced contact manifests in a high water contact angle $\left(>150^{\circ}\right)$ and low roll-off angle $\left(<10^{\circ}\right)$. Surface morphology can also confer benefits such as resisting bacterial attachment ${ }^{11}$ and 
protein fouling $^{12}$ and resisting platelet adhesion to reduce thrombosis. ${ }^{8}$

However, the usefulness of such surfaces is compromised by their gradual deterioration under exposure to various flow conditions. The loss of SHP properties can be categorized into chemical, physical, and recoverable degradation ${ }^{13}$. Chemical degradation occurs through the detachment of covalently attached self-assembled monolayers or by surface fouling (biofouling or chemical contamination). Physical degradation refers to the changes in surface roughness induced by physical damage (such as scratching, material removal, or flattening), which reduces the amount of trapped air in the plastron layer. Recoverable degradation refers to the loss of the plastron layer by hydrostatics or solution in the liquid phase over time, which can be exacerbated by high pressure in the fluid ${ }^{14}$ or with the spatial temporal variability of pressures during turbulent flows. The exposure of materials to the physiological milieu in blood, in which water and reactive oxygen species inflict hydrolytic and oxidative damage, further increases susceptibility to degradation ${ }^{15-17}$. Degradation of SHP surfaces may also occur under exposure to harsh environments, such as in highly turbulent flows in blood pumps, where the surface structures and coatings may be eroded by high fluid shear stresses; alternatively, the air plastron may be displaced by high fluid impalement pressures.

Several studies have presented strategies to resist the physical and chemical degradation of SHP surfaces ${ }^{18}$. These typically involve the use of hierarchical structures and multiscale surface roughness. The hierarchical nature of these surfaces exploits the more stable microscale structures to protect the more fragile nanoscale structures, which confer nonwettability ${ }^{19}$. Hierarchical structures have been produced using techniques such as vapor-induced phase separation, nanosphere lithography, and carbon nanotube alignment ${ }^{20-22}$. However, as these carefully engineered surface structures are very thin, they are easily abraded or damaged, leading to limited lifetime of the surface functionality $^{13}$. An auxiliary strategy to circumvent the degradation of SHP surfaces by wear is the use of monolithic bulk materials ${ }^{10}$ so that abrasion of the surface layers will only expose materials of similar properties.

Regarding recoverable degradation, the air plastron on SHP surfaces is easily destroyed. The plastron can be dissolved into the fluid, a process that speeds up with higher flow rates ${ }^{23}$. It can also be removed via mechanical agitation in the form of vibrations, squeezing, and impact. This is exacerbated in particulate flows (such as blood) in which the flow-induced particle-plastron collision has been found to shorten the plastron lifespan by up to $50 \%{ }^{24}$. This can undermine the low wettability of the surfaces and remove their drag reduction capacity. Previous studies have presented innovations to address this limitation through plastron stabilization ${ }^{25}$ and plastron regeneration ${ }^{26}$, but it remains likely that they cannot withstand strong and abrasive flow conditions.

In this study, we propose a functional material with easily executable strategies that extend the functional durability of superhydrophobic slip flow. First, a porous superhydrophobic polytetrafluoroethylene (PTFE, Teflon) foam was used as the superhydrophobic material, which could support an air plastron on the surface, enabling fluid slip flow and resisting protein fouling. Second, bulk foam was used instead of a thin surface coating to enhance abrasion durability. This foam represents an improvement upon our previous implementation of pore pressure control for superhydrophobic materials ${ }^{27}$, which relied on surface silanization for hydrophobicity and has substantially limited durability. Third, pressurized air was supplied to the pores in the foam to resist fluid impalement and to replenish air plastron lost to the fluid through dissolution to maintain the slip flow and antifouling function of the plastron layer. PTFE was selected due to its biocompatibility, innate hydrophobicity and chemical inertness, which are instrumental for bloodcontacting surfaces to prevent eliciting an immune response $^{28}$. We show its superior functional durability via studies of flow drag, plastron stability, protein fouling, and chemical and mechanical durability. Finally, we evaluated the material for its hemocompatibility, which illustrates its suitability for use in blood-contacting medical devices.

\section{Materials and methods \\ Materials}

PTFE foam (POREX® ${ }^{\circledR}$ irtek $^{\mathrm{TM}}$ PTFE BM 50, Porex Technologies, Norwich, UK) sintered from microsized PTFE particles with an average pore size of $3 \mu \mathrm{m}$, air permeability of $40 \mathrm{~L} / \mathrm{hr} / \mathrm{cm}^{2}$ (at a pressure gradient of 70 mbar), water entry pressure of 200 mbar and thickness of $2 \mathrm{~mm}$ was used as the base material. Porcine blood samples were ordered from the SingHealth Experimental Medical Centre (Singapore) with institutional approval and collected in sterile vacutainer tubes (Becton Dickinson, San Jose, CA, USA) containing K2 ethylenediaminetetraacetic acid (EDTA, $3 \mathrm{~mL}$ ) to prevent clotting. The MAK 115 Hemoglobin Assay Kit was purchased from Sigma-Aldrich (MO, USA). Serum FPA and sC5b-9 ELISA kits were purchased from Cloud-Clone Corp. (HO, USA). For the hemolysis assay, nitrile rubber (ARsoft, Alliance Rubber Products, Penang, Malaysia) and polydimethylsiloxane (PDMS, 1:10 mixing ratio, Sylgard 184, Dow Corning, MI, USA) were chosen as the positive and negative controls, respectively. For the other tests, the positive and negative controls were provided by the respective assay kits. Fluorescein isothiocyanateconjugated bovine serum albumin (FITC-BSA) powder, hydrochloric acid (35-38wt\%, $\mathrm{pH} 3.0)$ and sodium 
hydroxide (10 M in DI water, $\mathrm{pH} 12.0)$ were purchased from Sigma-Aldrich (MO, USA).

\section{Preparation and characterization of sanded PTFE foam}

We adopted a porous SHP material described in previous literature $\mathrm{e}^{29,30}$. The SHP PTFE foam was prepared by manual sanding with 400-grit aluminum oxide sandpaper (average particle diameter of $23 \mu \mathrm{m}$ ) for $2 \mathrm{~min}$ with moderate force; this preparation method was hypothesized to produce a surface roughness similar in magnitude to the grit sizes (which we confirmed from surface profiling). A prolonged sanding time and increased force applied were not expected to yield any changes in the surface wettability. The PTFE was sanded parallel to the flow direction in the drag reduction experiments to increase the drag reduction performance of the material ${ }^{30}$. Subsequently, the sanded foam was immersed in 99\% ethanol solution and cleaned by ultrasonication for $15 \mathrm{~min}$ to dislodge and remove any debris from the surface, and it was then dried at $90^{\circ} \mathrm{C}$ for $30 \mathrm{~min}$. This cleaning step was necessary to remove impurities that can act as condensation nuclei for water vapor or clog the pores of the material ${ }^{31}$.

The surface morphologies of the porous medium were imaged by scanning electron microscopy (SEM, JSM6701F, JEOL, Japan) after gold sputtering ( $20 \mathrm{~nm}$ thickness). The surface roughness (standard deviation of the surface topology) was measured using a profilometer (NanoMap-PS, aep Technology, Santa Clara, USA) at a vertical resolution of $1 \mu \mathrm{m}$. The wettability of the prepared samples was characterized by the contact angle (CA) and roll-off angle (RA). CA was measured using the sessile drop method with a $10 \mu \mathrm{L}$ drop of deionized (DI) water or blood. RA was measured using the tilting method by placing a $10 \mu \mathrm{L}$ droplet on a horizontally placed sample and recording the angle of tilt at which the droplet started to slide off. All CA and RA data were averaged over measurements at 3 different locations on the PTFE samples. The samples were mounted on the stage of a micromanipulator (M3301R, Prime Bioscience, Singapore), and images were captured with a high-definition camera (XCAM1080PHA, ToupTek, China).

\section{Hemocompatibility}

Hemocompatibility tests were conducted to evaluate the interaction of the superhydrophobic materials when in contact with blood. The hemocompatibility tests were selected according to ISO 10993-4:2017(E) (guidelines on Biological evaluation of medical devices-Part 4: Selection of tests for interactions with blood $)^{32}$, which included tests for hemolysis, coagulability, and complement system activation. For each test, whole blood was diluted 10 times in $1 \mathrm{X}$ PBS (Biowest, MO, USA) to $\sim 10 \mathrm{mg} / \mathrm{mL}$ as per the protocol of the ASTM International Standard on
Assessment of Hemolytic Properties of Material. Seven milliliters of diluted blood was added to $21 \mathrm{~cm}^{2}$ of the tested material. The mixture of diluted blood and cast material was incubated for $180 \mathrm{~min}$ at $37^{\circ} \mathrm{C}$, and the tubes were gently inverted every $30 \mathrm{~min}$. At the end of the incubation, the blood mixture was transferred to new centrifuge tubes and centrifuged at $800 \times g$ for $15 \mathrm{~min}$.

ELISA tests were performed according to the manufacturers' protocols. Photometric measurements were acquired with a spectrophotometer (SkanIt, Thermo Fisher Scientific, MA, USA). The hemolysis level was quantified based on the total plasma cell-free hemoglobin content measured using a hemoglobin assay kit. Serum FPA and sC5b-9 were measured using their respective ELISA kits.

\section{Protein adsorption}

Protein adsorption tests were conducted to simulate the fouling of the PTFE surface under prolonged exposure to blood. Albumin was selected as the fouling agent because it is the most abundant protein constituent in plasma ${ }^{33}$. FITC-BSA was reconstituted in 1X PBS to a concentration of $5 \mathrm{mg} / \mathrm{mL}$. Then, $10 \times 10 \mathrm{~mm}$ squares of the sanded PTFE foams and control nonporous PTFE sheets (RS Components, Singapore) were mounted on the base of a 24-well microplate (Corning, NY, USA). Next, $1.5 \mathrm{~mL}$ of FITC-BSA solution was added to each well and incubated at room temperature for 0,30 , and $60 \mathrm{~min}$. The incubated PTFE samples were washed twice with $1 \mathrm{X}$ PBS and mounted in a new 24-well microplate for protein adsorption quantification. The amount of absorbed albumin was quantified based on fluorescence intensity measured by epi-illumination (excitation/emission: 490/ $520 \mathrm{~nm}$ ) on an inverted microscope (Olympus IX71, Olympus, Japan) at $\times 4$ magnification.

\section{Functional durability tests}

The sanded PTFE samples were subjected to three different durability tests to ascertain the resilience and robustness of the material under harsh conditions. Performance impairment was evaluated from CA and RA measurements prior to and after the tests.

Acidity and alkalinity durability. The sample was separately immersed in a strong acid (hydrochloric acid, $\mathrm{pH}$ 3.0) and base (sodium hydroxide, $\mathrm{pH} 12.0$ ) for $5 \mathrm{~min}$ to evaluate its durability in strongly acidic or basic environments. The samples were cleaned with $99 \%$ ethanol by ultrasonication and dried at $80^{\circ} \mathrm{C}$ for $30 \mathrm{~min}$ prior to wettability measurements.

Water jetting. The material was jetted with a stream of water to assess its resistance to high shear stress. Strips of the sanded PTFE foams $(35 \times 14 \mathrm{~mm})$ were attached onto an acrylic frame with a needle gas inlet to supply pressurized nitrogen $\left(\mathrm{N}_{2}\right)$ gas through the pores of the PTFE material. The assembly was then mounted on a fixed frame, and a 
water jet was directed at the PTFE sample at a speed of $3.5 \mathrm{~m} / \mathrm{s}$ for either $60 \mathrm{~min}$ or $5 \mathrm{~h}$. This corresponds to an estimated shear stress of $20 \mathrm{kPa}$, which is consistent with the shear stress in a standard centrifugal blood pump design under average flow conditions (18 $\mathrm{kPa}$ at $2880 \mathrm{RPM}$ when adjusted for blood viscosity) ${ }^{34}$. We compared the effect of jetting on the samples with and without the supply of $\mathrm{N}_{2}$ gas at $100 \mathrm{kPa}$. The samples were dried at $80^{\circ} \mathrm{C}$ for $30 \mathrm{~min}$ prior to the wettability measurements.

Abrasion resistance. The mechanical durability of the PTFE samples was evaluated through an abrasion test. Strips of the sanded PTFE foams $(30 \times 14 \mathrm{~mm})$ were pasted onto an acrylic block that weighed $39.11 \mathrm{~g}$ and placed on top of the rough surface of \#400 aluminum oxide sandpaper. The PTFE foam was dragged with a velocity of $\sim 6 \mathrm{~cm} / \mathrm{s}$ for $10 \mathrm{~cm}$ to abrade the samples. These samples were subjected to 30,60 , and 90 abrasion cycles, followed by ultrasonic cleaning with $99 \%$ ethanol to dislodge loose particles and subsequent drying at $80^{\circ} \mathrm{C}$ for $30 \mathrm{~min}$.

\section{Drag reduction performance of sanded PTFE foam}

Open jetting study. The drag reduction performance was evaluated by the angle jetting test, following our previously published protocol ${ }^{10,27}$. In a similar setup as the water jetting test, the PTFE foam assembly was mounted onto a unidirectional linear ball-bearing slider that was connected to a force sensor (resolution: $0.1 \mathrm{mN}$; capacity: $980.0 \mathrm{mN}$ ), which allowed the imposed frictional forces on the PTFE assembly to be measured. High-speed water was jetted onto the porous PTFE assembly (nozzle diameter: $2.5 \mathrm{~mm}$; incidence angle: $20^{\circ}$ ) at varying flow velocities $(200-650 \mathrm{~mm} / \mathrm{s})$ monitored using a flow sensor (ME $10 \mathrm{PXN}$, Transonic, NY, USA). The gas pressure supplied to the PTFE assembly was regulated by a pressure controller (OB1 MK3 Pressure Controller, Elveflow, Paris, France). As the sample surface was partially covered by the jetted water stream, the actual air plastron pressure at the liquid-solid interface was expected to be markedly smaller than the set pressure due to air leakage through the porous areas not in contact with the jet. As a proof of concept, experiments were performed to compare the drag reduction of the sanded PTFE with and without a $30 \mathrm{kPa}$ gas supply against an acrylic control sample. The same tests were repeated on albumin-fouled PTFE samples to investigate the impact of protein fouling on the material superhydrophobicity. The drag reduction capacity of the materials was quantified in terms of the acrylic control by calculating the $\%$ drag reduction, given by the following equation:

$$
\% \text { drag reduction }=\frac{F_{C}-F_{T}}{F_{C}} \times 100 \%
$$

where $F_{C}$ and $F_{T}$ refer to the forces experienced by the control and tested samples, respectively.
Closed loop system. The drag reduction was further evaluated in a closed loop flow system to simulate its integration into an extracorporeal flow system. Two sample assemblies, each comprising a $14 \times 100 \mathrm{~mm}$ strip of sanded PTFE mounted on an acrylic frame with 3 backing gas inlets for pressurized gas supply, were fitted into the closed loop chamber (Supplementary Fig. S1). The PTFE samples were connected in series with a water reservoir, a centrifugal pump (BIO-MEDICUS 540 BIO-CONSOLE, Medtronic, MN, USA) and a flow meter (T402, Transonic, NY, USA). Two pressure gauges (HYA-101A, 0-100 kPa/ $0.05 \%$ accuracy, Hua Nan Instrument, China) were connected immediately upstream and downstream of the sample to measure the pressure drop across the superhydrophobic material. A computer-controlled gas regulator (OB1 MK3 Pressure Controller, Elveflow, Paris, France) connected to a $\mathrm{N}_{2}$ cylinder and a vacuum pump was capable of supplying pressures ranging from -100 to $100 \mathrm{kPa}$. The pressure drop $(\Delta P)$ was recorded across a range of supplied gas pressures $(-20 \mathrm{kPa}$ to $10 \mathrm{kPa})$ for 3 pump speeds (500, 1000, 1500 RPM). The Cassie-Baxterto-Wenzel transition occurred when water breached the material and flowed into transparent tubing connected to the gas inlet. Drag reduction experiments were conducted with varying pump speeds (500-3000 RPM). The drag reduction was estimated from the $\Delta P$ reduction at each flow rate calculated using the following relation:

$$
\% \Delta \text { Preduction }=\frac{\Delta P_{w}-\Delta P_{C}}{\Delta P_{w}} \times 100 \%
$$

where $\Delta P_{w}$ and $\Delta P_{c}$ refer to the pressure drop across the test sample in the Wenzel and Cassie states, respectively. Flow stability was measured based on the change in the flow resistance $(R=\Delta P / Q)$ normalized by the initial resistance:

$$
\text { normalized } \Delta R=\frac{R_{t}-R_{i}}{R_{i}}
$$

where $R_{t}$ and $R_{i}$ refer to the instantaneous and initial resistances, respectively.

\section{Droplet impact}

Droplet impact tests were conducted to investigate the ability of the PTFE foam to achieve droplet bouncing on its irregular surface with nonuniform porosities supplemented with air plastron pressure control. Impact tests were performed by dropping a $21 \mu \mathrm{l}$ droplet of DI water (diameter $D_{0} \approx 3.4 \mathrm{~mm}$ ) onto the PTFE sample surface from a height $h_{0}$ of $32.8 \mathrm{~mm}$. This corresponds to a Weber number of $30 \quad\left(W e=\rho V_{0}^{2} D_{0} / \gamma, V_{0}=\sqrt{2 g h_{0}}\right.$, where $\rho$ and $\gamma$ refer to the density $\left(998 \mathrm{~kg} \mathrm{~m}^{-3}\right)$ and surface tension $\left(72.8 \mathrm{mN} \mathrm{m}^{-1}\right)$ of water, respectively, and 
$g$ is the gravitational acceleration constant). Impact dynamics were monitored using a monochrome highspeed camera (frame rate: 10,000 fps; FASTCAM Mini AX200, Photron, Tokyo, Japan) by measuring the contact time $t_{c}$ (duration between droplet contact and complete rebound, averaged over 3 measurements) and observing the droplet deformation. The droplet bouncing ability of the PTFE foam was compared against that of nonporous PTFE sheets (RS Components, Singapore) and standard microscope glass slides (VWR, PA, USA).

\section{Statistical analysis}

Statistical analyses were performed using a commercially available software (OriginPro 2017, OriginLab, MA, USA), and all statistical significance levels were tested at the $5 \%$ level. The results are presented as the mean \pm standard deviation. Unpaired Student's $t$ tests (one-tailed) were used to assess the significant differences in the wettability between the control and test samples for the tests on sanding, hemocompatibility and functional durability. One-way analysis of variance (ANOVA) was used to assess the statistical significance between the 3 time points for the protein adsorption tests and across the range of Reynolds numbers and gas pressures in the closed loop drag reduction test. Where applicable, post hoc analyses were performed with Tukey's honestly significant difference test.

\section{Results and discussion}

\section{Characterization of sanded PTFE foam}

Surface morphology and superhydrophobicity. The pristine PTFE samples were fabricated from microparticles sintered together to form a porous foam bulk material with an average pore size of $3 \mu \mathrm{m}$, as reported by the manufacturer. SEM images revealed wide, nonuniform porosities with a measured surface roughness of $24.97 \mu \mathrm{m}$, corroborating the reported specification (Fig. 1 (b)). After sanding, we observed narrower and more uniform grooves that were imparted by the grits of the sandpaper (Fig. 1(c)). Profilometry revealed a reduced surface roughness of $6.35 \mu \mathrm{m}$, corresponding to the smoothening of the surface post sanding. The porous geometry produces discrete three-phase (solid-air-liquid) contact lines in the form of "point" contacts, which have been empirically demonstrated to have extremely low surface adhesion due to the trapping of larger air plastrons ${ }^{35}$, and the narrowing of the grooves and reduced randomness after sanding improve the stability of the air plastrons ${ }^{36}$.

Surface wettability measured using the sessile drop technique revealed that even before sanding, the PTFE foam itself was nearly superhydrophobic with a CA of $150.96 \pm 3.74^{\circ}$ and RA of $15.68 \pm 2.43^{\circ}$. This superhydrophobicity is attributed to the inherent micro/ nanoscale structure of the bulk PTFE foam, which was derived from the sintering process of the foam in tandem with the intrinsically low surface energy of the $\mathrm{PTFE}^{37}$. After sanding with 400-grit sandpaper, the CA increased slightly to $156.43 \pm 3.59^{\circ}$, while the RA decreased drastically to $6.41 \pm 1.65^{\circ}$, which satisfied the conditions of superhydrophobicity (Fig. 1(a)). To ascertain its suitability for use in blood-contacting medical devices, we also quantified the CA and RA for blood using the same protocol (Fig. 2(a)). While the CA was comparably high $\left(151.90 \pm 0.46^{\circ}\right)$, the RA was above the criteria for superhydrophobicity $\left(20.77 \pm 0.76^{\circ}\right)$, which nonetheless
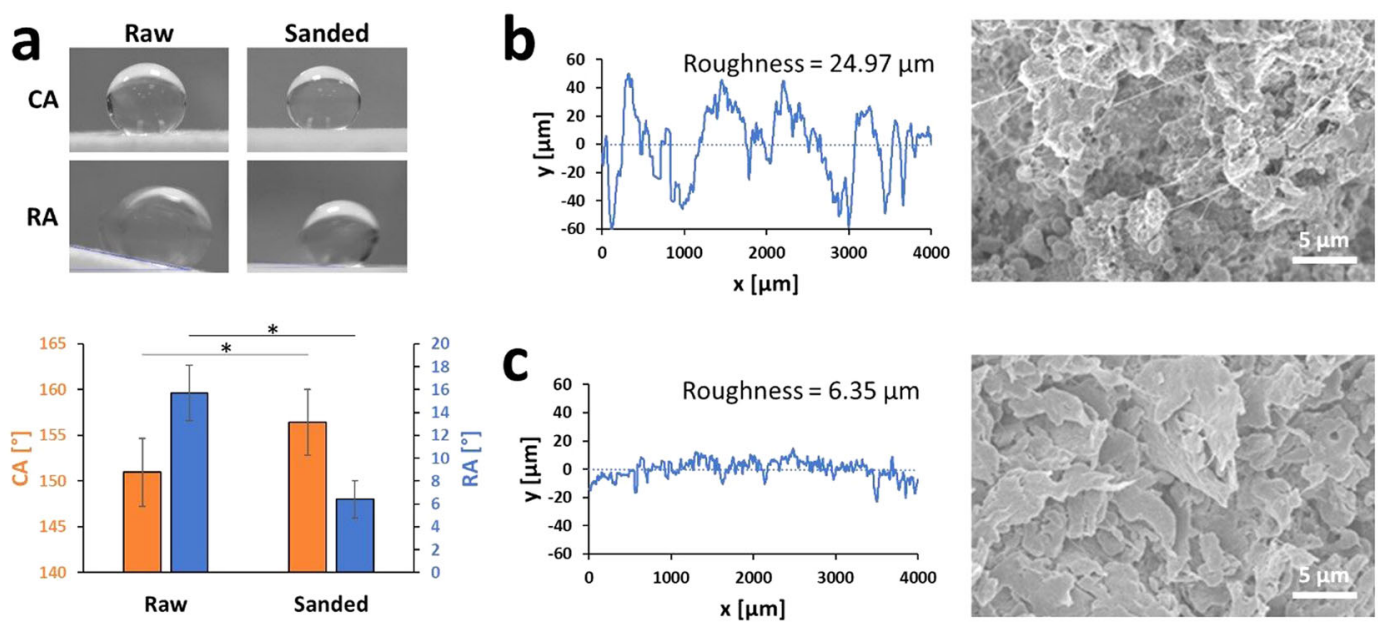

Fig. 1 Characterization of material superhydrophobicity and surface topology. a Contact angle (CA) and roll-off angle (RA) measured by sessile drop goniometry $\left.{ }^{*} p<0.05\right)$. b, c Surface roughness and the corresponding SEM images of the raw and sanded PTFE samples, respectively. The sanded sample displayed the presence of hierarchical microstructures that support the presence of an air plastron for reduced solid-liquid contact. 
illustrates that the surface is still highly hydrophobic to blood. This result may be attributed to the interaction of plasma proteins with the material surface exacerbated by local increases in protein concentration in the vicinity of the contact line driven by evaporation-induced capillary flow $^{38}$. The randomness of the sanded surface, as demonstrated from profilometry and electron microscopy, has previously been shown to impart higher superhydrophobicity in general, albeit at the cost of lower plastron stability; these surfaces typically lose their superhydrophobicity at higher hydraulic pressures ${ }^{36,39}$. This result supports the need for an auxiliary system to augment the plastron stability to widen the range of operating flow conditions.

Hemocompatibility. Hemocompatibility tests were performed to ascertain the suitability of the material for use in direct contact with blood. First, hemolysis assays were performed to quantify the rupturing of red blood cells caused by contact with the material ${ }^{40}$. The sanded PTFE had a comparable level of hemolysis $(3.75 \pm 3.12 \%)$ to the negative sample (PDMS, $3.31 \pm 1.47 \%$ ), hence demonstrating its low hemolytic capacity (Fig. 2(b), left). Second, fibrinopeptide A (FPA) was measured to establish the coagulability of blood in contact with the material. FPA is a byproduct of the polymerization reaction of fibrinogen to fibrin, which is an indication of coagulation activation $^{41}$. The sanded PTFE foam yielded low levels of FPA $(2.18 \pm 0.09 \mathrm{ng} / \mathrm{mL})$, which were similar to the negative control $(2.03 \pm 0.14 \mathrm{ng} / \mathrm{mL})$, hence demonstrating that the material causes negligible clotting of blood (Fig. 2(b), middle). Finally, soluble C5b-9 levels were tested to determine the activity of the innate complement reaction of the immune system. Soluble C5b-9 is a terminal complement activation product that, at high levels, signifies increased activation of the innate complement immune system $^{42}$. The sanded PTFE foam showed a low production of sC5b-9 $(35.46 \pm 2.70 \mathrm{ng} / \mathrm{mL})$, which was comparable to the negative control $(37.07 \pm 2.07 \mathrm{ng} / \mathrm{mL})$, hence indicating that PTFE is unlikely to elicit an immunologic response (Fig. 2(b), right).

Protein adsorption. Figure 2(c) shows the amount of albumin protein adsorbed onto the sanded PTFE foam (porous) compared against a nonporous PTFE sheet over a 1-h interval. On the nonporous samples, the amount of adsorbed albumin increased monotonically with the incubation time, with most of the albumin absorption occurring in the initial $30 \mathrm{~min}$ of incubation $(130.34 \pm 8.98$ arbitrary units, A.U.). Further incubation for up to $60 \mathrm{~min}$ slightly increased the amount of absorbed albumin to $136.65 \pm 7.69$ A.U. However, the sanded, porous PTFE samples showed only minor levels of protein adsorption over the 1-h incubation period ( $2.74 \pm 1.35$ A.U., $7.28 \pm$ 6.31 A.U., $6.75 \pm 4.31$ A.U. at 0,30 and $60 \mathrm{~min}$, respectively, $p » 0.05)$. To verify the role of the plastron in blocking protein adsorption, we forced the PTFE samples into the Wenzel state by submerging them in PBS and
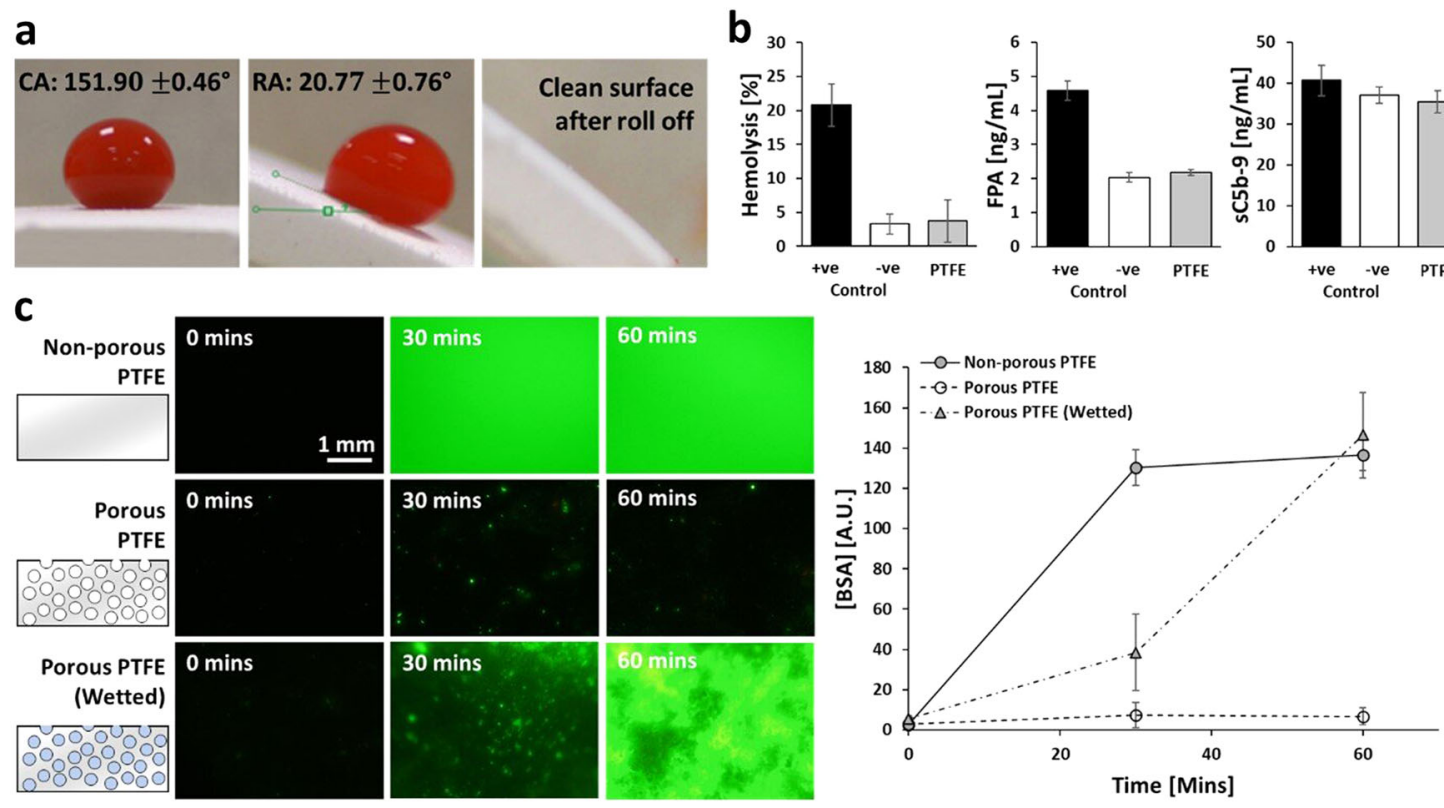

Fig. 2 Characterization of material interaction with blood. a CA and RA measured by sessile drop goniometry. The blood droplet rolls off, leaving a clean surface. b Hemocompatibility tests for (left to right) hemolysis, coagulability and complement immune system activation. "+ve" and "-ve" refer to the positive and negative controls, respectively. c Concentration of bovine serum albumin (BSA) adsorbed on the PTFE surface over a 1-h incubation period. The sanded, porous PTFE was compared against nonporous PTFE for its protein adsorption susceptibility. 
a
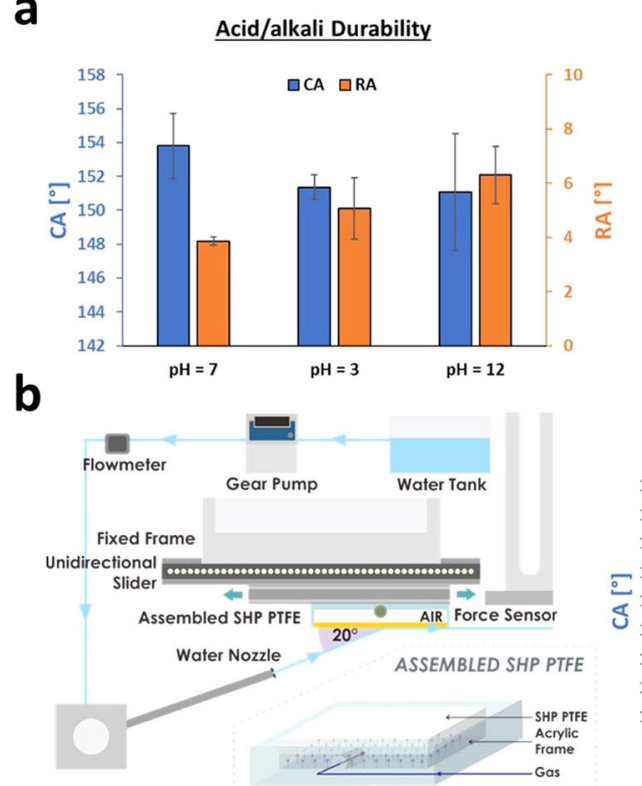

C
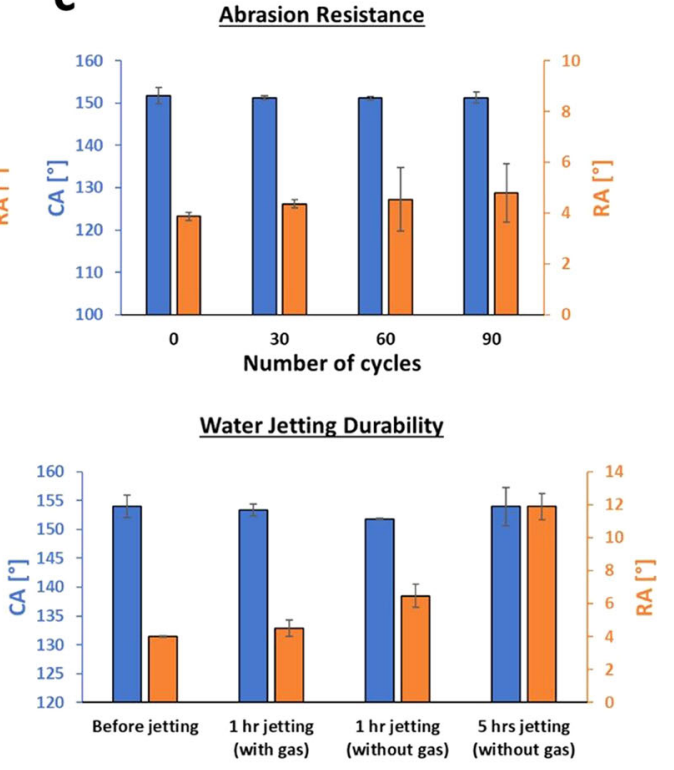

Fig. 3 Characterization of material functional durability. a CA and RA measurements of the PTFE surface after incubation in solutions of varying $\mathrm{pH}$. $\mathbf{b}$ (left) Schematic diagram of the setup for the water jetting test used for the functional durability and drag reduction tests. A stream of water is jetted onto the PTFE sample assembly with a backing gas supply. (right) Wettability characterization of the PTFE surface before and after jetting, comparing the effects of pressurized gas supply. c Abrasion resistance evaluation of the PTFE material after varying the number of abrasion cycles with \#400 grit sandpaper.

compressing them under high pressure $(\sim 1000 \mathrm{kPa})$, which displaced the air within the plastron layer in the surface asperities before the protein adsorption tests were performed. The displacement of air resulted in significant protein adsorption over the 1-h duration comparable to the nonporous samples $(5.60 \pm 1.47$ A.U., $38.54 \pm 18.91$ A. U., $146.42 \pm 21.32$ A.U. at 0,30 and $60 \mathrm{~min}$, respectively, $p<0.05)$. These results demonstrate that without active air plastron support, protein readily adsorbs onto the material surface when in contact with blood plasma, which has detrimental effects on the surface wettability. The absence of protein adsorption on the porous PTFE samples demonstrates the beneficial shielding effect of the air plastron. This result is consistent with those of previous studies that suggested that the air plastron reduces liquid-solid contact and hence reduces the protein interaction with the material ${ }^{43,44}$. Maintaining the durability of the plastron layer can thus be an antifouling strategy. This is particularly instrumental in reducing the adsorption of plasma proteins such as fibrinogen, fibronectin and von Willebrand factor, which trigger the intrinsic pathway of coagulation, hence reducing the incidence of deviceinduced thrombosis ${ }^{2,3}$.

Functional durability. The results of the functional durability tests are presented in Fig. 3. In the first test, the material was immersed in strong acid and alkali solutions to investigate the possible degradation of the superhydrophobicity (Fig. 3(a)). No statistically significant differences were measured in the $C A$ after either the acidic or alkaline treatment $(p>0.05)$. RA increased in both solutions but remained lower than $10^{\circ}$, hence demonstrating the preservation of the superhydrophobicity. This result is consistent with that of a previous study in which the PTFE-based material was incubated within a $\mathrm{pH}$ range of 1 to 14 for $24 \mathrm{~h}$ and registered minimal changes in the measured CA and RA, both of which remained within the superhydrophobic range ${ }^{45}$. This finding may be attributed to broad chemical compatibility and resistance of PTFE to most acids, alcohols, and solvents stemming from the high bond strength of the constituent $\mathrm{C}-\mathrm{C}$ and $\mathrm{C}-\mathrm{F}$ bonds ${ }^{28,46}$.

In the second test, the material was jetted with a high velocity stream of water $(3.5 \mathrm{~m} / \mathrm{s})$ to subject the material to high and abrasive fluid shear stress (Fig. 3(b)). No significant differences in CA and RA were measured in the PTFE samples with pressurized gas supplied compared to the sanded PTFE foam. The PTFE samples without gas supplied had a slightly reduced CA of $151.80^{\circ}$ and higher RA of $6.46^{\circ}$, but nonetheless still maintained its superhydrophobicity. The RA increased further to $11.88^{\circ}$ after jetting for a total of $5 \mathrm{~h}$, which may be due to the gradual protein fouling of the surface due to prolonged environmental exposure. The mounted PTFE foam supplied with $100 \mathrm{kPa}$ of $\mathrm{N}_{2}$ gas was less susceptible to shear-induced 
changes in the wettability. This decreased susceptibility is most likely due to the synergistic effects of pore pressure stabilization of the air plastron and reduced material erosion due to decreased solid-liquid interaction.

Mechanical durability is a critical consideration for biomaterials, as it governs the functional lifespan of the material. Structurally weak materials may also suffer from excessive wear and consequently lead to debris dislodgement ${ }^{47}$. In the final test, the material was abraded with \#400 grit sandpaper to evaluate the effects of mechanical abrasion on the surface wettability. There were no observable changes in CA with an increasing number of abrasion cycles $\left(\sim 151^{\circ}\right)$, while the RA was shown to increase slightly from $3.87 \pm 0.15^{\circ}$ to $4.80 \pm 1.15^{\circ}$ after 90 abrasion cycles (Fig. 3 (c)). The excellent abrasion resistance is due primarily to the use of a bulk PTFE foam monolith compared to a thin coating, which would have eroded easily with abrasion, which has been demonstrated in previous studies. Zimmerman et al. ${ }^{48}$, Xiu et al. ${ }^{49}$, and Zhu et al..$^{50,51}$ subjected their SHP materials to similarly harsh abrasion conditions and reported varying degrees of reduction of their materials' wettability stemming from destruction of fragile surface nanostructures or chemical coatings. This material contrasts with the SHP polymer composite material developed by Wang et al. ${ }^{52}$ which, similar to ours, was monolithic in nature and withstood mechanical abrasion with no appreciable change in CA or sliding angle.

\section{Drag reduction functionality of sanded PTFE foam}

Blood cells that flow through blood-contacting medical devices are exposed to artificial stresses resulting from collision with foreign surfaces, high fluid shear stresses and turbulence ${ }^{4,5}$. We have previously demonstrated the reduction in hemolysis with the use of a nearsuperhydrophobic surface through the reduction of fluid shear stresses with slip flow ${ }^{53}$. In this study, we demonstrated the drag reduction capacity of our sanded PTFE foam to establish its ability to reduce blood damage in foreign systems. We selected a broad range of test conditions to highlight the robustness of the material and its suitability of use across a wide range of ex vivo or extracorporeal applications. For example, the lower range of Reynolds numbers can be applicable to dialysis blood circuits $^{54,55}$ and in ECMO oxygenators ${ }^{56,57}$, while the higher range $(>2300)$ at which flow turbulence is present is relevant to blood pumps such as that in ECMO and the heart lung machine ${ }^{58}$ and in the cannula of such extracorporeal applications ${ }^{59-61}$.

Open jetting study. The drag reduction capability of the sanded PTFE foam was evaluated by open water jetting.
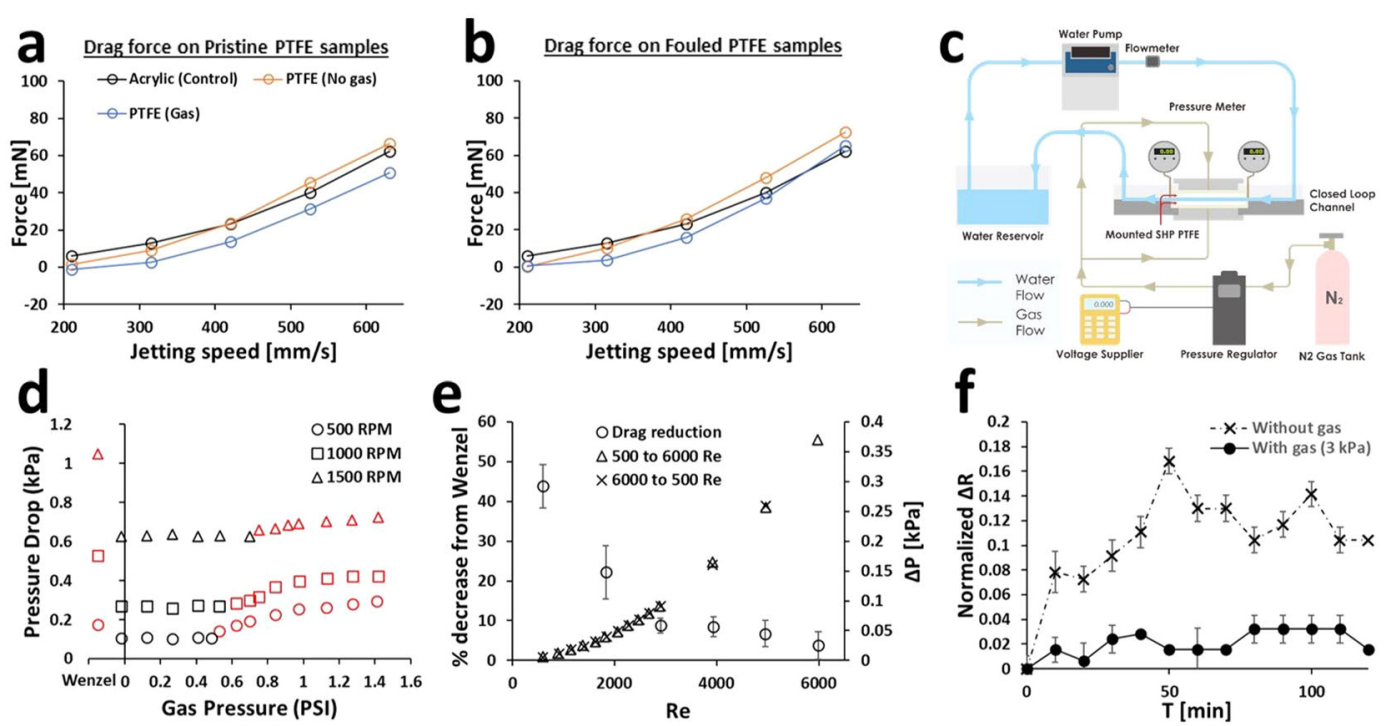

Fig. 4 Evaluation of the drag reduction performance of the sanded PTFE foam with pneumatic active plastron stabilization. a Results of the open jetting drag reduction test. The PTFE foam (with and without gas supply) was compared against the acrylic control in terms of the measured drag force. $\mathbf{b}$ The open jetting test repeated with an albumin-fouled PTFE sample, which showed generally inferior drag reduction capabilities after protein fouling. A pressurized gas supply was able to restore the drag reducing function of the material. c Schematic diagram of the setup for the closed loop drag reduction test. The test sample was connected in series to a centrifugal pump, water reservoir and flowmeter. Pressure sensors immediately upstream and downstream of the test sample measured the pressure drop across the sample. $\mathbf{d}$ Pressure drop measured across the test samples with varying gas pressures under different pump speeds. The red symbols indicate data points corresponding to the Wenzel state (left) or the bubbling regime (right). (e) Drag reduction with varying Reynolds numbers, comparing the pressure drop between the Cassie-Baxter (while supplied with $3 \mathrm{kPa}$ of N2 gas) and Wenzel states. $\mathbf{f}$ Normalized $\triangle \mathrm{R}$ monitored over $120 \mathrm{~min}$ under different sample conditions (nonfouled vs in situ fouling; with gas supply vs without gas supply). 
The drag force experienced by the PTFE foam with and without pressurized gas supplied to its pores at each jetting speed is shown in Fig. 4(a). In these experiments, we used a flat and smooth piece of acrylic as our no-slip control. The results showed that without a pressurized gas supply, drag reduction from the control could be achieved at a lower jetting speed. However, at higher jetting speeds above $400 \mathrm{~mm} / \mathrm{s}$, a higher drag than that of the control was registered. This higher drag was due to high fluid impalement pressure that enabled water penetration through the pores, resulting in a Cassie-Baxter-to-Wenzel transition, and this wet, rough surface, having a higher roughness than that of the control, would naturally experience higher drag forces under turbulent flows. With pressurized gas supply, however, a large drag reduction was observed at lower jetting velocities but decreased monotonically with increasing jetting speeds. With the pressurized gas supplied to the PTFE material, the stabilized air plastron was capable of repelling water from the gap between the surface asperities, hence preserving the slip flow on the material surface. From these results, it is evident that the sanded PTFE foam already had intrinsic drag reduction capabilities. However, high impalement pressures can destabilize the air plastron and force the material surface into the Wenzel state, hence increasing the skin friction. This shortcoming can be circumvented by increasing the pore pressure in the porous material with the supply of pressurized gas, which counters the high impalement pressure and stabilizes the air plastron.

Prolonged exposure of any biomaterial to blood leads to protein fouling, which can modify the wettability of the surface. Proteins in blood plasma bind readily to superhydrophobic surfaces either through direct interactions of hydrophobic regions on the protein surface or through denaturation of the protein, which exposes underlying hydrophobic residues ${ }^{62-64}$. The protein binding increases the surface energy of the PTFE surface, which favors interaction with water, leading to a loss of the superhydrophobic properties and drag reduction capabilities, and can greatly limit the functional durability of the material. To determine the possible implications of protein fouling on the PTFE surface wettability and drag reduction performance, we performed the open jetting test on PTFE samples incubated in an albumin solution for $60 \mathrm{~min}$ (Fig. 4(b)). With albumin fouling, drag forces generally increase, and the drag reduction capabilities decrease. Without gas pressure provided to the pores, the Cassie-Baxter-to-Wenzel transition point was reduced to a lower jetting velocity, demonstrating that the air pores were easier to breach. However, when the gas pressure was controlled to a lower jetting speed, the fouled sample could still achieve drag reductions up to $50 \%$ after incubation in albumin solutions for $60 \mathrm{~min}$. This result demonstrates that the strategy of using a porous superhydrophobic material with pressurized air plastron is a good strategy to maintain drag reduction functionality despite protein adsorption.

Closed loop system. Drag reduction performance was further evaluated in a closed loop flow system that mimicked an extracorporeal flow loop (Fig. 4(c)). Pressure sensors positioned immediately up- and downstream of the test samples allowed for the measurement of the pressure drop across the test samples, which provided a quantitative measure of the drag resistance of the segment. Figure 4(d) shows the measured pressure drop across a range of supplied gas pressures at different flow rates. There were three main phases for the pressure drop across the range of gas pressures used. At gas pressures above a critical value, bubbling occurred inside the channel due to the excessive $\mathrm{N}_{2}$ flow through the samples. This bubbling resulted in excessive drag generated as a result of the bubbles obstructing the flow. The critical gas pressure required to induce bubbling increased with increasing fluid pressure, which was induced with a higher flow rate in our flow loop. Below this threshold pressure, the pressure drop remained consistently low across a wide range of gas pressures. In this region, the sample surface is in the Cassie-Baxter regime, which could be verified through CA measurements directly after experimentation. When we supplied a transient high negative gauge pressure $(-30 \mathrm{kPa})$ to the air pores, we could induce a breach of water into the air pores and a transition to the Wenzel state, which resulted in wetting of the surface and increased drag.

The comparison between the drag forces during the Cassie-Baxter and Wenzel states in Fig. 4(d) could be used to determine the amount of drag reduction offered by the superhydrophobic slip flow on the material and is plotted in Fig. 4(e). A pressure drop reduction of up to $\sim 50 \%$ was registered at the lowest flow rates within the laminar flow regime but decreased nonlinearly with increasing flow rates. At Reynolds numbers exceeding 3000 up to a maximum tested value of 6000 (corresponding to a pump speed of 3000 RPM), the flow was likely turbulent, and the drag reduction was reduced to less than $10 \%$. One explanation for this behavior could be that the high spatial and temporal variability of pressures of the turbulent flow caused localized disturbances of the fluid meniscus on the material surface, disrupting the slip flow properties. When the flow was gradually increased from a Reynolds number of 540 to a Reynolds number of 6000 and then decreased back to 540, drag reduction results were repeatably obtained, as shown in Fig. 4(e). This result illustrates the stability of the plastron even under high-shear and turbulent conditions.

In addition to the stabilization of the air plastron against fluid impalement, active pneumatic control has an auxiliary function. It has been previously reported that the 
rate at which the air plastron is lost through gas diffusion across the liquid-air meniscus is significant ${ }^{65,66}$. It is responsible for the shrinkage or disappearance of the air plastron, decreasing the functional lifespan of superhydrophobic materials without an active gas supply. As our material adopts an active gas supply to sustain an air plastron, any plastron lost through dissolution is compensated by the inflow of gas. The longevity of superhydrophobicity is ensured as the influence of gas dissolution is removed. This phenomenon was demonstrated through temporal monitoring of the drag reduction performance of the sanded PTFE material with and without gas supply over $2 \mathrm{~h}$. In the sample without gas supply, an impervious barrier was placed behind the sample. The results in Fig. 4(f) show a significant increase in the normalized $\Delta \mathrm{R}(10-20 \%)$ across the sample without the gas supply over the 2 -h duration, indicating an increase in the fluid drag and corroborating our hypothesis and previous literature. However, the sample with an active gas supply of $3 \mathrm{kPa}$ displayed a stable and low pressure drop over the test duration.

To demonstrate the versatility of this pneumatic approach in controlling the surface wettability, we tested the ability to deterministically convert between the Cassie-Baxter and Wenzel states by controlling the gas pressure. By cyclically switching the gas supply pressure between -30 and $30 \mathrm{kPa}$, we were able to achieve switchable wettability between the superhydrophobic and hydrophilic states, with a $10 \%$ drag reduction observed when changing from the hydrophilic to hydrophobic states (Supplementary Fig. S2). This drag reduction may be enhanced by optimizing the water expulsion and gas flow through the material to achieve better uniformity in plastron control across the material surface. This feature may be capitalized on for deterministic flow valving and control in extracorporeal circuits or for deep cleaning of blood-contacting surfaces.

\section{Droplet impact test}

Droplet impact onto the sanded PTFE foam surface with and without active plastron pressure control followed the conventional droplet bouncing dynamics (Fig. 5 (a) and (b)). Upon contact with the material surface, the droplet first spread to a maximum diameter at $\sim 5.6 \mathrm{~ms}$, retracted with the formation of a Worthington jet, and then fully rebounded and detached from the surface at $t_{c}{ }^{67,68}$. This bouncing phenomenon is attributed to the rectification of the stored capillary energy in the droplet upon penetration of the surface asperities into kinetic energy to drive the upward motion of the droplet ${ }^{69,70}$. The $t_{c}$ was observed to decrease by $\sim 25 \%$ from $30.27 \pm 2.79 \mathrm{~ms}$ to $22.67 \pm 0.57 \mathrm{~ms}$ with 1 bar of supplied $\mathrm{N}_{2}$ gas (Fig. 5(e)), which is due to the additional energy supplied by the pore air pressure beneath the droplet ${ }^{27}$. We noted that due to air leakage through the porous surface not in contact with the droplet, the air plastron pressure at the liquid-solid

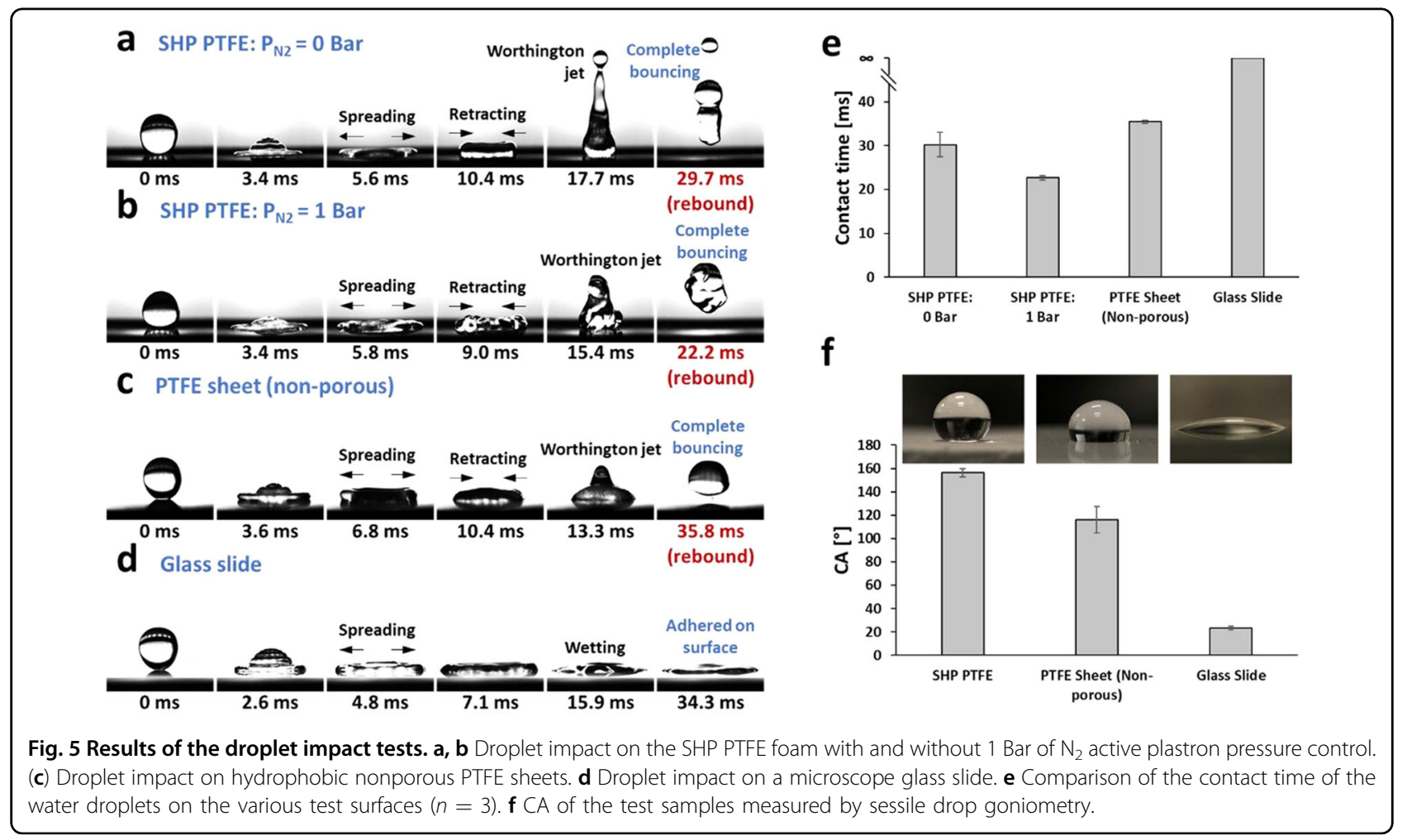


interface would have been smaller than 1 Bar. Nonetheless, the pressure may be controlled by facile adjustment of the $\mathrm{N}_{2}$ pressure to modulate the droplet bouncing dynamics.

For comparison, we also conducted droplet impact tests on hydrophobic nonporous PTFE sheets and hydrophilic glass slides with measured CAs of $116.00 \pm 11.17^{\circ}$ and $23.25 \pm 1.57^{\circ}$, respectively (Fig. 5(f)). Droplet bouncing was also observed for the nonporous PTFE sheets (Fig. 5 (c)), albeit with a longer $t_{c}$ of $35.50 \pm 0.36 \mathrm{~ms}$. This result was expected since in the absence of surface roughness, the bouncing is driven purely by transient conversion of translational kinetic energy into elastic potential energy and back, some of which is dissipated by viscous squeezeflow in the lubrication layer between the droplet and the surface $^{71,72}$. No bouncing was observed on the hydrophilic glass slides. No retraction of the droplet was observed after spreading, and the liquid wetted and adhered to the surface due to the high surface energy of the glass surface (Fig. 5(d)). This feature of our PTFE foam demonstrates the resistance of our material to fluid impalement.

\section{Conclusion}

In this study, we presented a robust method of air plastron stabilization through active pneumatic air plastron control to oppose the fluid impalement pressure. This technique was integrated into a porous SHP PTFE foam that could be easily prepared. The material exhibited excellent water- and blood-repelling capabilities and functional durability and could achieve significant drag reduction (up to 50\%). We combined this strategy with that of using a bulk foam material, which provided high abrasion durability to the material. Furthermore, we demonstrated that the maintenance of the air plastron layer ensured the stability of the surface wettability through replenishment of the air as well as protein shielding even after prolonged use in a flow loop. Our strategy can easily be integrated into existing bloodcontacting medical devices, such as extracorporeal systems, and presents great promise in alleviating common associated hemolytic or thrombotic events to improve patient outcomes.

\section{Acknowledgements}

This work was supported by the Singapore National Medical Research Council (NMRC)/Open Fund-Individual Research Grant (OFIRG)/0060/2017.

\section{Author details \\ ${ }^{1}$ Department of Biomedical Engineering, National University of Singapore, Singapore, Singapore. ${ }^{2}$ Department of Civil \& Environmental Engineering, University of Alberta, Edmonton, AB, Canada. ${ }^{3}$ Department of Chemical Engineering, University of Waterloo, Waterloo, ON, Canada. ${ }^{4}$ Department of Bioengineering, Imperial College London, London, UK}

\section{Competing interests}

The authors declare no competing interests.

\section{Publisher's note}

Springer Nature remains neutral with regard to jurisdictional claims in published maps and institutional affiliations.

Supplementary information The online version contains supplementary material available at https://doi.org/10.1038/s41427-021-00325-9.

Received: 22 February 2021 Revised: 30 June 2021 Accepted: 9 July 2021. Published online: 13 August 2021

\section{References}

1. Jaffer, I. H. \& Weitz, J. I. The blood compatibility challenge. Part 1: bloodcontacting medical devices: The scope of the problem. Acta Biomaterialia $\mathbf{9 4}$, 2-10 (2019).

2. Jaffer, I., Fredenburgh, J., Hirsh, J. \& Weitz, J. Medical device-induced thrombosis: what causes it and how can we prevent it? J. Thrombosis. Haemost. 13, S72-S81 (2015).

3. Leslie, D. C. et al. A bioinspired omniphobic surface coating on medical devices prevents thrombosis and biofouling. Nat. Biotechnol. 32, 1134-1140 (2014).

4. Olia, S. E., Maul, T. M., Antaki, J. F. \& Kameneva, M. V. Mechanical blood trauma in assisted circulation: sublethal RBC damage preceding hemolysis. Int. J. Artif. Organs 39, 150-159 (2016).

5. Kameneva, M. V. et al. Effects of turbulent stresses on mechanical hemolysis: experimental and computational analysis. ASAIO J. (Am. Soc. Artif. Intern. Organs: 1992) 50, 418 (2004).

6. Levin, A. P. et al. Watchful waiting in continuous-flow left ventricular assist device patients with ongoing hemolysis is associated with an increased risk for cerebrovascular accident or death. Circulation: Heart Fail. 9, e002896 (2016).

7. Hoshian, S., Kankuri, E., Ras, R. H., Franssila, S. \& Jokinen, V. Water and blood repellent flexible tubes. Sci. Rep. 7, 1-8 (2017).

8. Jokinen, V., Kankuri, E., Hoshian, S., Franssila, S. \& Ras, R. H. Superhydrophobic blood-repellent surfaces. Adv. Mater. 30, 1705104 (2018).

9. Salimi, E. Superhydrophobic blood-compatible surfaces: state of the art. Int. J. Polymeric Mater. Polymeric Biomater. 69, 363-372 (2019).

10. Li, Z. et al. Durable, flexible, superhydrophobic and blood-repelling surfaces for use in medical blood pumps. J. Mater. Chem. B 6, 6225-6233 (2018).

11. Zhang, X., Wang, L. \& Levänen, E. Superhydrophobic surfaces for the reduction of bacterial adhesion. Rsc Adv. 3, 12003-12020 (2013).

12. Koc, Y. et al. Nano-scale superhydrophobicity: suppression of protein adsorption and promotion of flow-induced detachment. Lab a Chip 8, 582-586 (2008).

13. Crick, C. R. Approaches for Evaluating and Engineering Resilient Superhydrophobic Materials. In Superhydrophobic Surfaces: Fabrications to Practical Applications. IntechOpen, 11-38 (2018).

14. Samaha, M. A., Vahedi Tafreshi, H. \& Gad-el-Hak, M. Sustainability of superhydrophobicity under pressure. Phys. Fluids 24, 112103 (2012).

15. Tamariz, E. \& Rios-Ramírez, A. Biodegradation of medical purpose polymeric materials and their impact on biocompatibility. In Biodegradation-Life of Science Croatia. Intech, 1-29 (2013).

16. Boland, E. L., Shine, R., Kelly, N., Sweeney, C. A. \& McHugh, P. E. A review of material degradation modelling for the analysis and design of bioabsorbable stents. Ann. Biomed. Eng. 44, 341-356 (2016).

17. Azevedo, H. S. \& Reis, R. L. Understanding the enzymatic degradation of biodegradable polymers and strategies to control their degradation rate. In Biodegradable Systems in Tissue Engineering and Regenerative Medicine. CRC Press, 178-197 (2005).

18. Mortazavi, V. \& Khonsari, M. On the degradation of superhydrophobic surfaces: a review. Wear 372, 145-157 (2017).

19. Verho, T. et al. Mechanically durable superhydrophobic surfaces. Adv. Mater. 23, 673-678 (2011).

20. Zhao, N. et al. Superhydrophobic surface from vapor-induced phase separation of copolymer micellar solution. Macromolecules 38, 8996-8999 (2005).

21. Xie, Q. et al. Facile creation of a bionic super-hydrophobic block copolymer surface. Adv. Mater. 16, 1830-1833 (2004).

22. Li, S. et al. Super-hydrophobicity of large-area honeycomb-like aligned carbon nanotubes. J. Phys. Chem. B 106, 9274-9276 (2002).

23. Samaha, M. A., Tafreshi, H. V. \& Gad-el-Hak, M. Influence of flow on longevity of superhydrophobic coatings. Langmuir 28, 9759-9766 (2012). 
24. Hokmabad, B. V. \& Ghaemi, S. Effect of flow and particle-plastron collision on the longevity of superhydrophobicity. Sci. Rep. 7, 1-10 (2017).

25. Teisala, $H$. et al. Ultrafast processing of hierarchical nanotexture for a transparent superamphiphobic coating with extremely low roll-off angle and high impalement pressure. Adv. Mater. 30, 1706529 (2018).

26. Panchanathan, D., Rajappan, A., Varanasi, K. K. \& McKinley, G. H. Plastron regeneration on submerged superhydrophobic surfaces using in situ gas generation by chemical reaction. ACS Appl. Mater. interfaces 10, 33684-33692 (2018).

27. Li, Z., Marlena, J., Pranantyo, D., Nguyen, B. L. \& Yap, C. H. A porous superhydrophobic surface with active air plastron control for drag reduction and fluid impalement resistance. J. Mater. Chem. A 7, 16387-16396 (2019).

28. Ebnesajjad, S. Properties of Fluoropolymers. In Fluoroplastics, volume 2: Melt processible fluoropolymers-the definitive user's guide and data book. William Andrew, 475-563 (2015)

29. Nilsson, M. A., Daniello, R. J. \& Rothstein, J. P. A novel and inexpensive technique for creating superhydrophobic surfaces using Teflon and sandpaper. J. Phys. D: Appl. Phys. 43, 045301 (2010).

30. Song, D., Daniello, R. J. \& Rothstein, J. P. Drag reduction using superhydrophobic sanded Teflon surfaces. Exp. Fluids 55, 1783 (2014).

31. LV, P. et al. Symmetric and asymmetric meniscus collapse in wetting transition on submerged structured surfaces. Langmuir 31, 1248-1254 (2015).

32. International Organization for Standardization. Guidelines on Biological evaluation of medical devices - Part 4: Selection of tests for interactions with blood (ISO/DIS Standard No. 10993-4:2017). (2017).

33. Schenk, S., Schoenhals, G. J., de Souza, G. \& Mann, M. A high confidence, manually validated human blood plasma protein reference set. BMC Med. Genomics 1, 41 (2008).

34. Cao, W., Jia, Z. \& Zhang, Q. Near-wall flow characteristics of a centrifugal impeller with low specific speed. Processes 7, 514 (2019).

35. Lai, Y. et al. Designing superhydrophobic porous nanostructures with tunable water adhesion. Adv. Mater. 21, 3799-3803 (2009).

36. Lee, C., Choi, C.-H. \& Kim, C.-J. Superhydrophobic drag reduction in laminar flows: a critical review. Exp. Fluids 57, 176 (2016).

37. Ebnesajjad, S. Surface treatment of fluoropolymers for adhesion. In Fluoroplastics, volume 2: Melt processible fluoropolymers-the definitive user's guide and data book. William Andrew, 564-586 (2015).

38. Sobac, B. \& Brutin, D. Desiccation of a sessile drop of blood: cracks, folds formation and delamination. Colloids Surf. A: Physicochem. Eng. Asp. 448, 34-44 (2014).

39. Samaha, M. A., Vahedi Tafreshi, H. \& Gad-el-Hak, M. Modeling drag reduction and meniscus stability of superhydrophobic surfaces comprised of random roughness. Phys. fluids 23, 012001 (2011).

40. Gupta, R. C. Blood and bone marrow toxicity biomarkers. In Biomarkers in toxicology. Academic Press, 401-412 (2019).

41. Weisel, J. W. \& Litvinov, R. I. Mechanisms of fibrin polymerization and clinical implications. Blood. 121, 1712-1719 (2013).

42. Rus, H., Cudrici, C. \& Niculescu, F. The role of the complement system in innate immunity. Immunologic Res. 33, 103-112 (2005).

43. Huang, Q. et al. Role of trapped air in the formation of cell-and-protein micropatterns on superhydrophobic/superhydrophilic microtemplated surfaces. Biomaterials 33, 8213-8220 (2012).

44. Leibner, E. S. et al. Superhydrophobic effect on the adsorption of human serum albumin. Acta biomaterialia 5, 1389-1398 (2009).

45. Zhuang, A. et al. Transparent superhydrophobic PTFE films via one-step aerosol assisted chemical vapor deposition. RSC Adv. 7, 29275-29283 (2017).

46. McKeen, Led.. in The Effect of Sterilization on Plastics and Elastomers (Third Edition) William Andrew Publishing, 261-276 (2012).

47. Savio lii, J., Overcamp, L. \& Black, J. Size and shape of biomaterial wear debris. Clin. Mater. 15, 101-147 (1994).

48. Zimmermann, J., Reifler, F. A., Fortunato, G., Gerhardt, L. C. \& Seeger, S. A simple, one-step approach to durable and robust superhydrophobic textiles. Adv. Funct. Mater. 18, 3662-3669 (2008).
49. Xiu, Y., Liu, Y., Hess, D. W. \& Wong, C. Mechanically robust superhydrophobicity on hierarchically structured Si surfaces. Nanotechnology 21, 155705 (2010).

50. Zhu, X. et al. Robust superhydrophobic surfaces with mechanical durability and easy repairability. J. Mater. Chem. 21, 15793-15797 (2011).

51. Zhu, X. et al. Facile fabrication of a superhydrophobic fabric with mechanical stability and easy-repairability. J. Colloid Interface Sci. 380, 182-186 (2012)

52. Wang, F., Lei, S., Ou, J., Xue, M. \& Li, W. Superhydrophobic surfaces with excellent mechanical durability and easy repairability. Appl. Surf. Sci. 276 397-400 (2013).

53. Lai, C. Q., Shen, J. C. W., Cheng, W. C. W. \& Yap, C. H. A near-superhydrophobic surface reduces hemolysis of blood flow in tubes. RSC Adv. 6, 62451-62459 (2016).

54. Kim, S. et al. Diffusive silicon nanopore membranes for hemodialysis applications. Plos One 11, e0159526 (2016).

55. Weitzel, W. F. et al. Analysis of novel geometry-independent method for dialysis access pressure-flow monitoring. Theor. Biol. Med. Model. 5, 1-12 (2008).

56. Bartlett, R. H. Physiology of extracorporeal gas exchange. Compr. Physiol. 10 879-891 (2011).

57. Weissman, M. \& Mockros, L. Oxygen and carbon dioxide transfer in membrane oxygenators. Med. Biol. Eng. 7, 169-184 (1969).

58. Berg, N., Fuchs, L. \& Wittberg, L. P. Flow characteristics and coherent structures in a centrifugal blood pump. Flow., Turbulence Combust. 102, 469-483 (2019)

59. Gu, K., Zhang, Y., Gao, B., Chang, Y. \& Zeng, Y. Hemodynamic differences between central ECMO and peripheral ECMO: a primary CFD study. Med Sci. Monit. 22, 717-726, https://doi.org/10.12659/msm.895831 (2016).

60. Van Meurs, K. P., Mikesell, G., Seale, W. R., Short, B. L. \& Rivera, O. Maximum blood flow rates for arterial cannulae used in neonatal ECMO. ASAIO Trans. $\mathbf{3 6}$ M679-681 (1990).

61. Ganushchak, Y. M., Kurniawati, E. R., Maessen, J. G. \& Weerwind, P. W. Peripheral cannulae selection for veno-arterial extracorporeal life support: a paradox. Perfusion 35, 331-337 (2020).

62. Falde, E. J., Yohe, S. T., Colson, Y. L. \& Grinstaff, M. W. Superhydrophobic materials for biomedical applications. Biomaterials 104, 87-103 (2016).

63. Shen, J.-W., Wu, T., Wang, Q. \& Kang, Y. Induced stepwise conformational change of human serum albumin on carbon nanotube surfaces. Biomaterials 29, 3847-3855 (2008).

64. Ishak, N. F., Hashim, N. A., Othman, M. H. D., Monash, P. \& Zuki, F. M. Recent progress in the hydrophilic modification of alumina membranes for protein separation and purification. Ceram. Int. 43, 915-925 (2017).

65. Xu, M., Sun, G. \& Kim, C.-J. C. Wetting dynamics study of underwater superhydrophobic surfaces through direct meniscus visualization. In 2014 IEEE 27th International Conference on Micro Electro Mechanical Systems (MEMS). 668-671 (2014).

66. Xiang, Y. et al. Ultimate stable underwater superhydrophobic state. Phys. Rev. Lett. 119, 134501 (2017)

67. Richard, D., Clanet, C. \& Quéré, D. Contact time of a bouncing drop. Nature 417, 811-811 (2002).

68. Reyssat, M., Pépin, A., Marty, F., Chen, Y. \& Quéré, D. Bouncing transitions on microtextured materials. EPL (Europhys. Lett.) 74, 306 (2006).

69. Yu, X., Zhang, Y., Hu, R. \& Luo, X. Water droplet bouncing dynamics. Nano Energy 81, 105647 (2020).

70. Bartolo, D. et al. Bouncing or sticky droplets: Impalement transitions on superhydrophobic micropatterned surfaces. EPL (Europhys. Lett.) 74, 299 (2006).

71. De Ruiter, J., Lagraauw, R., Van Den Ende, D. \& Mugele, F. Wettabilityindependent bouncing on flat surfaces mediated by thin air films. Nat. Phys. 11, 48-53 (2015).

72. Gilet, T. \& Bush, J. W. Droplets bouncing on a wet, inclined surface. Phys. Fluids 24, 122103 (2012). 